\title{
Prognostic Significance of Sentinel Lymph Node Mapping in Merkel Cell Carcinoma: Systematic Review and Meta-Analysis of Prognostic Studies
}

\author{
Ramin Sadeghi, ${ }^{1}$ Zohreh Adinehpoor, ${ }^{1}$ Masoud Maleki, ${ }^{2}$ Babak Fallahi, ${ }^{3}$ \\ Luca Giovanella, ${ }^{4}$ and Giorgio Tregliaa ${ }^{4}$ \\ ${ }^{1}$ Nuclear Medicine Research Center, Mashhad University of Medical Sciences, Mashhad 9176699199, Iran \\ ${ }^{2}$ Cutaneous Leishmaniasis Research Center, Mashhad University of Medical Sciences, Mashhad 9137913316, Iran \\ ${ }^{3}$ Research Institute for Nuclear Medicine, Tehran University of Medical Sciences, Tehran 1411713137, Iran \\ ${ }^{4}$ Department of Nuclear Medicine and PET/CT Center, Oncology Institute of Southern Switzerland, Via Ospedale 12, \\ 6500 Bellinzona, Switzerland
}

Correspondence should be addressed to Giorgio Treglia; giorgiomednuc@libero.it

Received 28 February 2014; Accepted 13 May 2014; Published 26 May 2014

Academic Editor: Rei Shibata

Copyright (C) 2014 Ramin Sadeghi et al. This is an open access article distributed under the Creative Commons Attribution License, which permits unrestricted use, distribution, and reproduction in any medium, provided the original work is properly cited.

\begin{abstract}
Aim. To assess through a systematic review and meta-analysis of the literature the prognostic implication of sentinel lymph node mapping in Merkel cell carcinoma (MCC). Materials and Methods. PubMed and SCOPUS databases were searched by using "Merkel AND sentinel" as keywords. All studies with prognostic information regarding SLN mapping in cN0 MCC patients were included. Hazard ratio (HR) for overall survival (OS) and disease free survival (DFS) was used as effect size. Results. SLN biopsy predicted better DFS and OS as compared to the nodal observation in cN0 MCC patients (pooled HR for DFS: 1.61 (95\% CI: 1.05-2.46), $P=0.028$; pooled HR for OS: 1.08 (95\% CI: $0.55-2.10$ ), $P=0.8$ ). Pathologically negative SLN (SLN-) patients had better OS (pooled HR: 4.42 (95\% CI: 1.82-10.7), $P=0.0009$ ) and DFS (pooled HR: 2.58 (95\% CI: 1.78-3.73)) as compared to SLN+ patients. Conclusion. SLN mapping can provide strong prognostic information regarding OS and DFS in cN0 MCC patients. More importantly, SLN mapping can improve DFS and possibly OS in cN0 MCC patients as compared to nodal observation. As MCC is a rare tumor, large multicenter prospective studies are still needed to validate the survival benefit of SLN mapping.
\end{abstract}

\section{Introduction}

Merkel cell carcinoma (MCC) is a rare cutaneous neuroendocrine malignancy with high propensity for regional lymph node spread and recurrence [1]. Its origin is believed to be the primitive epidermal stem cells capable of epithelial or neuroendocrine differentiation [2]. This tumor is aggressive with high mortality and poor prognosis. However, the clinical course of MCC can be more aggressive in males, elderly patients, large tumors, and immunocompromised individuals $[3,4]$. One of the most important prognostic factors in clinically node negative (cN0) MCC patients is the presence of occult regional lymph nodal involvement $[2,4-6]$.
Sentinel lymph node (SLN) mapping is an important and accurate technique for regional lymph node staging in many solid tumors [7-9]. By identifying the first lymph node in a nodal basin that receives the lymph flow from a solid tumor, SLN biopsy allows for careful pathologic evaluation of one or a few SLNs (instead of the whole regional basin) that are most likely to be pathologically involved.

There are several studies which reported the prognostic importance of positive SLN in MCC. It is reported that patients with positive SLN have 3 times higher relapse rate and 2 times higher disease specific mortality as compared to negative SLN patients $[10,11]$. However, the literature is heterogeneous in this regard and several authors did not 
find statistical significant association between positive SLN pathology and recurrence or survival [4].

More importantly, a number of studies reported that patients with regional lymph node metastasis have better survival and lower recurrence when treated with lymphadenectomy or regional radiation therapy $[12,13]$. It seems that early detection of regional nodal involvement in MCC patients by SLN biopsy can improve survival due to the early start of more aggressive treatment plans in the disease course [14]. However, other studies showed no survival benefit by SLN mapping in MCC and no consensus has been reached in this regard [15].

In the current study, we aimed to assess through a systematic review and meta-analysis of the literature the prognostic implication of sentinel lymph node (SLN) pathological status in patients with MCC evaluating whether SLN mapping may improve survival in $\mathrm{cN} 0 \mathrm{MCC}$ patients.

\section{Material and Methods}

2.1. Search Strategy. We followed the PRISMA guidelines for performing the current systematic review and meta-analysis (http://www.prisma-statement.org/). We searched PubMed and SCOPUS databases using the following search algorithm: "merkel AND sentinel." The literature search was performed by two authors independently and the last search was done on January 2014 without language or time limit. The reference lists of the relevant studies were reviewed for possible missing citations.

2.2. Inclusion Criteria. Studies which met one of the following criteria were included in the systematic review:

(1) evaluation of prognostic value of pathologic involvement of SLN for determination of overall survival (OS) or disease free survival (DFS) in cN0 MCC patients;

(2) evaluation of the prognostic value of SLN mapping in cN0 MCC patients for improvement of OS or DFS.

In addition, we collected individual patient data from the reported cases in the literature with enough prognostic information (at least pathological involvement of the SLN and time to events such as death or recurrence should be reported) in order to perform an individual patient meta-analysis. Letters to the editor and review articles were excluded. Studies without enough prognostic information were also excluded. Two authors reviewed independently the retrieved articles. All discrepancies were resolved by the third author opinion. Possible duplicate publications were discussed and only the most recent reports were considered.

2.3. Data Abstraction. Two authors independently performed the data abstraction, and data on authors, affiliations, publication date, SLN mapping method, patient characteristics, and quality of the included studies were retrieved. The main effect size we used in the current analysis was hazard ratio (HR) of DFS and/or OS which were either extracted directly from the included studies or estimated from survival curves as recommended by Parmar et al. [20]. We reported pooled values with $95 \%$ confidence intervals $(95 \%$ CI). Oxford center for evidence-based medicine checklist for prognostic studies was used to assess the quality of the included studies [21].

2.4. Statistical Analyses. Dersimonian and Laird method (random effects model) was used to pool the HR among the studies [22]. The pooled results were expressed graphically by forest plots. Cochrane $Q$ test was used for heterogeneity evaluation ( $P<0.05$ was considered statistically significant). The inconsistency $\left(I^{2}\right)$ index was used to quantify the heterogeneity among the studies.

For publication bias evaluation, funnel plots and Egger's regression intercept were used [23]. All statistical analyses were performed by using Comprehensive Meta-analysis (version 2, Biostat Inc., USA) and SPSS (version 11.5, SPSS Inc., USA).

\section{Results}

Figure 1 shows the PRISMA flowchart of the study. Four studies (1417 patients) provided HR of DFS and/or OS for SLN mapping as compared to other regional treatments in cN0 MCC patients $[3,14,17,18]$. One of the included studies compared SLN mapping with all other treatments including clinical observation and lymph node dissection [3]. The other three compared the SLN mapping with observation in $\mathrm{cN} 0$ MCC patients.

Three studies (883 patients) provided HR of DFS and/or OS for pathologic condition of harvested SLN $[15,16,19]$.

Three studies had prognostic information based on the Surveillance, Epidemiology, and End Results (SEER) database $[4,14,16]$. One of them had duplicate information and it was excluded [4]; therefore two articles were included in the systematic review $[14,16]$.

The characteristics of the included studies and quality assessment are shown in Table 1.

Twenty-one studies including 172 patients had prognostic information regarding pathological condition of SLN $[10,24-$ 43]. Three studies had duplicate cases and were excluded [44-46]. We used cox regression model to analyze these cases and HR of OS and DFS for cN0 MCC patients with pathologically involved SLN as compared to pathologically noninvolved nodes were 6.13 (95\% CI: 1.97-19.07) and 2.25 (95\% CI: 1.16-4.33), respectively. Detailed survival analyses of these 172 patients are shown in Table 2.

3.1. Prognostic Importance of SLN Mapping versus Other Nodal Treatment Strategies. Quantitative synthesis is shown in Figure 2. Operative nodal staging with SLN biopsy predicted better DFS as compared to the nodal observation in $\mathrm{cN} 0$ MCC patients (pooled HR: 1.61 (95\% CI: 1.05-2.46), $P=$ 0.028 , Cochrane $Q$ value $\left.=2.36, P=0.3, I^{2}=15.3 \%\right)$. Nodal staging with SLN biopsy also predicted a slightly better OS, although the pooled HR (1.08 (95\% CI: 0.55-2.10), Cochrane 


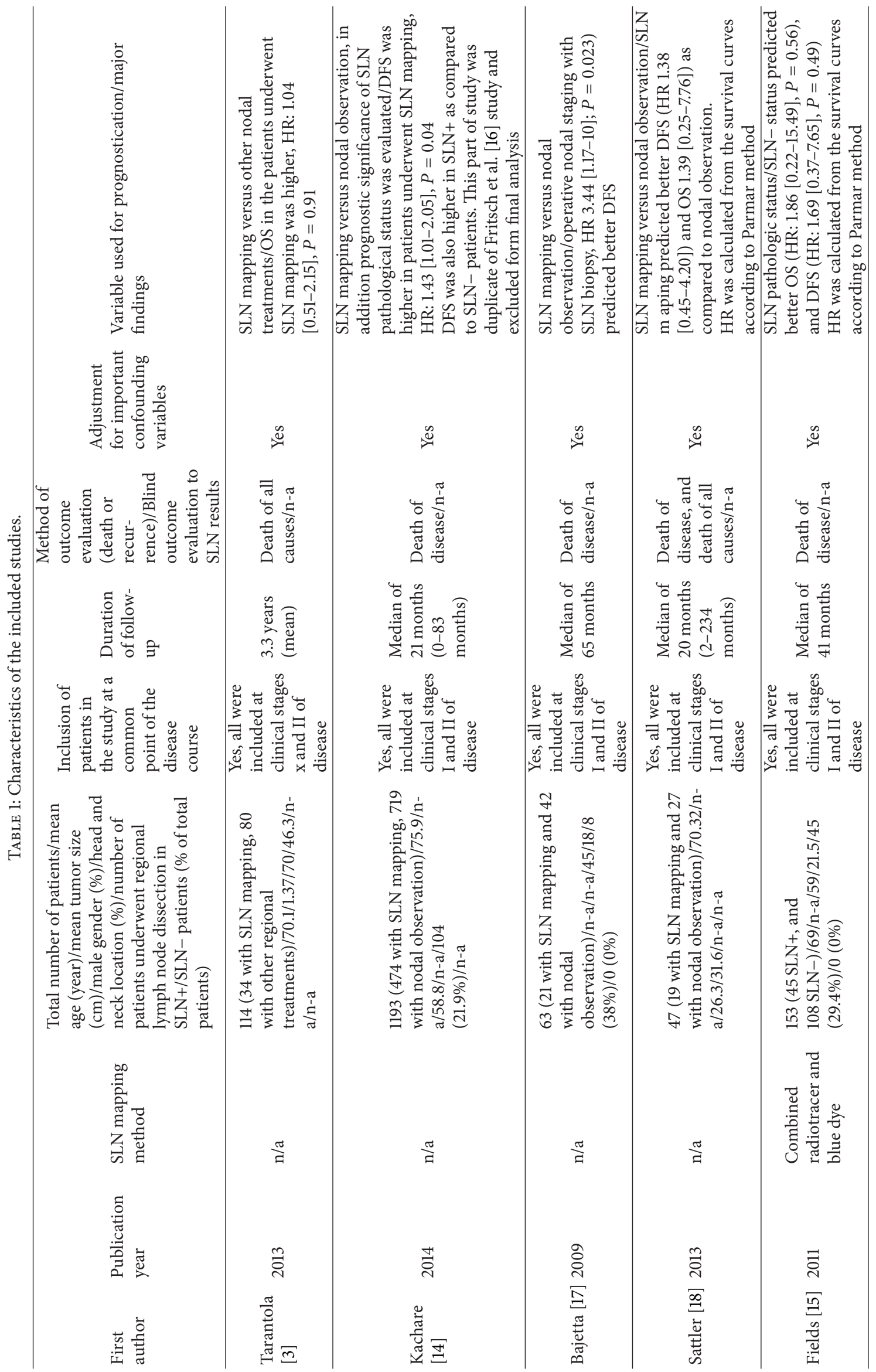




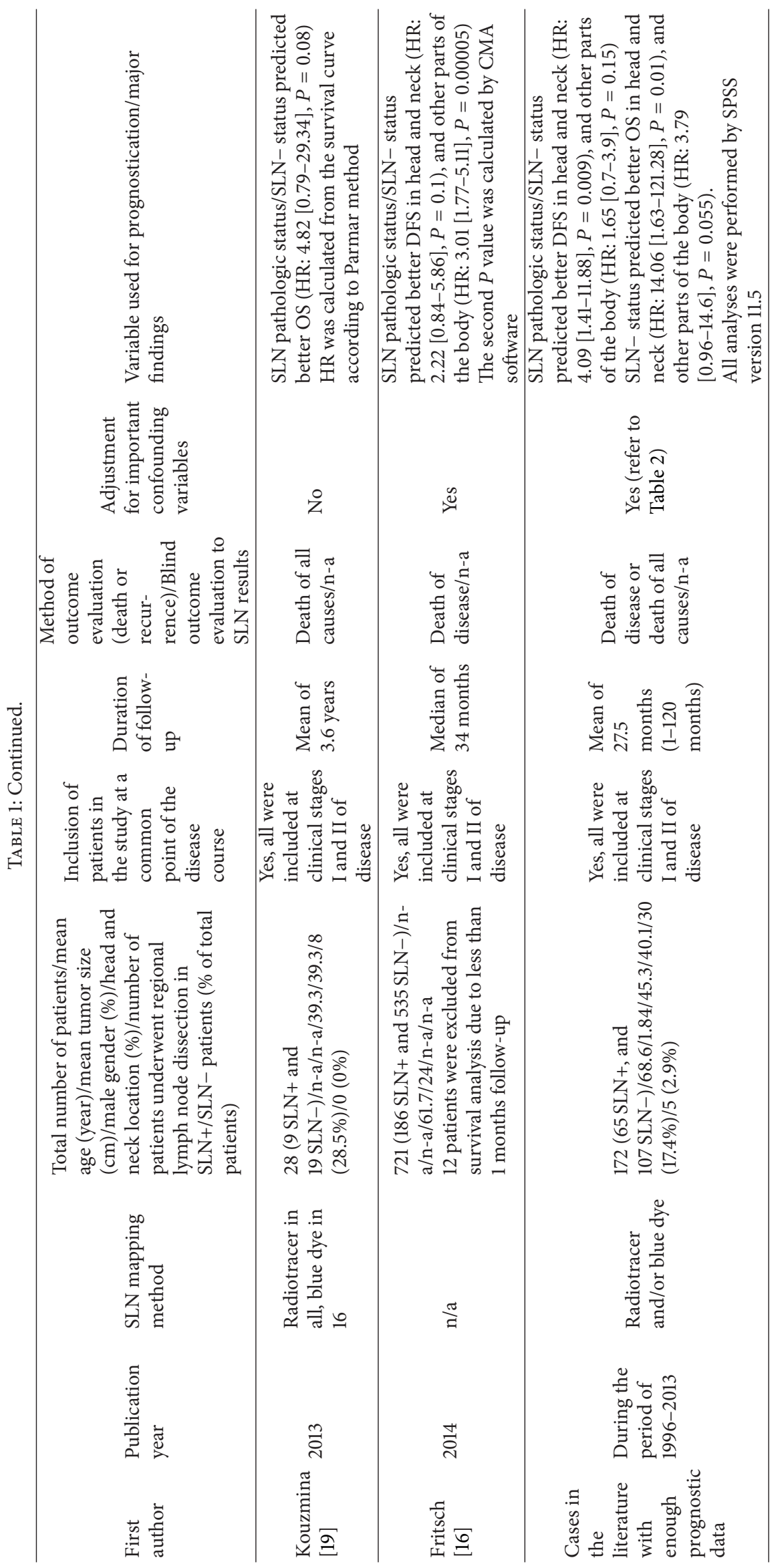




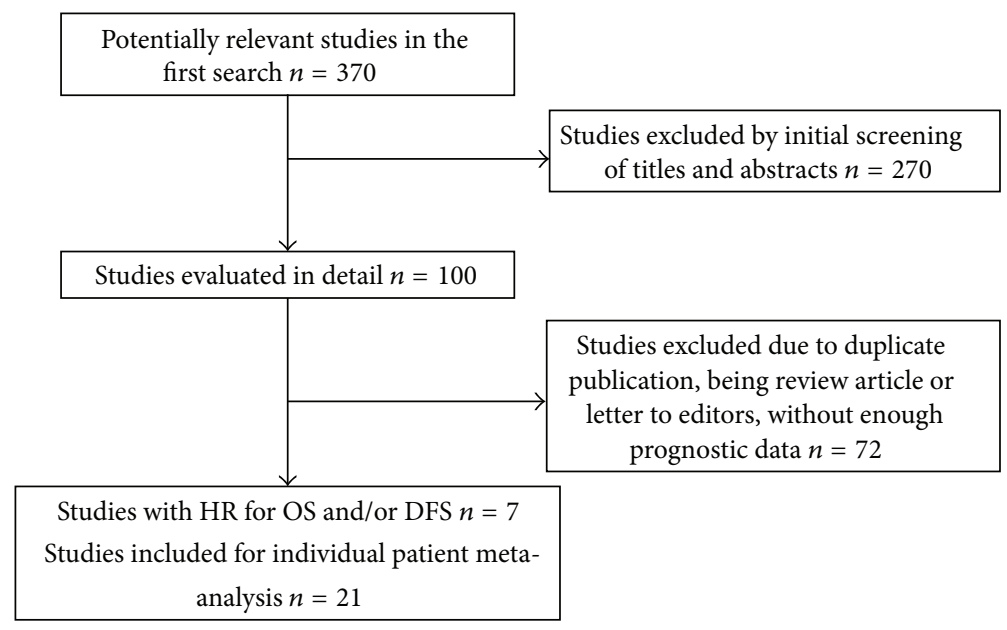

FIGURE 1: PRISMA flowchart of the study.

TABLE 2: Detailed survival analysis of the cases included in the individual patient analysis $(n=172)$.

\begin{tabular}{|c|c|c|c|c|c|}
\hline Factor & Number of patients & HR for OS [95\% CI] & $P$ value & HR for DFS $[95 \% \mathrm{CI}]$ & $P$ value \\
\hline Age & Mean 68.58 & $1.039[0.98-1.09]$ & 0.13 & $1.003[0.97-1.03]$ & 0.83 \\
\hline Tumor size & Mean 1.85 & $0.59[0.23-1.5]$ & 0.27 & $1.017[0.65-1.53]$ & 0.93 \\
\hline Gender & & & 0.212 & & 0.55 \\
\hline Male & 78 & $1.86[0.69-5.02]$ & & $1.23[0.62-2.44]$ & \\
\hline Female & 83 & Referent & & Referent & \\
\hline \multicolumn{6}{|c|}{ Regional nodal treatment } \\
\hline None & 100 & $0.17[0.04-0.72]$ & 0.16 & $0.28[0.1-0.78]$ & 0.015 \\
\hline Nodal dissection & 23 & $0.78[0.18-3.31]$ & 0.74 & $0.68[0.22-2.14]$ & 0.51 \\
\hline Radiotherapy & 37 & $0.66[0.11-3.99]$ & 0.65 & $0.37[0.1-1.29]$ & 0.12 \\
\hline Both & 12 & Referent & Referent & Referent & Referent \\
\hline \multicolumn{6}{|l|}{ Tumor location } \\
\hline Limbs & 95 & $0.95[0.33-2.71]$ & 0.93 & $0.73[0.37-1.46]$ & 0.38 \\
\hline Trunk & 8 & $2.08[0.24-17.52]$ & 0.46 & $1.95[0.56-6.77]$ & 0.29 \\
\hline Head and neck & 69 & Referent & Referent & Referent & Referent \\
\hline SLN status & & & 0.002 & & 0.015 \\
\hline Positive & 65 & $6.13[1.97-19.07]$ & & $2.25[1.16-4.33]$ & \\
\hline Negative & 107 & Referent & & Referent & \\
\hline
\end{tabular}

$Q$ value $\left.=0.093, P=0.76, I^{2}=0 \%\right)$ was not statistically significant $(P=0.8)$.

3.2. Prognostic Importance of SLN Pathologic Status for Prediction of DFS and OS. Quantitative synthesis is shown in Figure 3. Pathologically negative SLN (SLN-) patients had better OS (pooled HR: 4.42 (95\% CI: 1.82-10.7), $P=0.0009$, Cochrane $Q$ value $=1.8, P=0.61, I^{2}=0 \%$ ) and DFS (pooled HR: 2.58 (95\% CI: 1.78-3.73), $P=0.000001$, Cochrane $Q$ value $\left.=2.47, P=0.64, I^{2}=0 \%\right)$ as compared to SLN+ patients.

Funnel plots of OS and DFS meta-analyses are shown in Figure 4. Egger's regression intercepts for DFS and OS meta-analyses were $-0.79(P=0.52)$ and $0.82(P=0.76)$, respectively.

Subgroup analysis regarding location of MCC did not show any difference between head and neck and other parts of the body regarding prognostic importance of SLN pathologic status (pooled HR of DFS for head and neck and other parts of the body were 2.92 (95\% CI: $1.42-6), P=0.003$, and 2.54 (95\% CI: 1.62-4), $P=0.000048$, resp.).

\section{Discussion}

SLN mapping is an integral part of treatment in melanoma and breast cancer patients [47-49]. SLN mapping has been used to evaluate MCC for a long time and prognostic significance of this technique has been assessed in several reports. Recently, data of SEER database have been published regarding prognostic importance of SLN mapping in MCC, increasing our understanding in this regard.

In the current study, we reviewed the medical literature for two purposes: (1) to determine the prognostic importance 


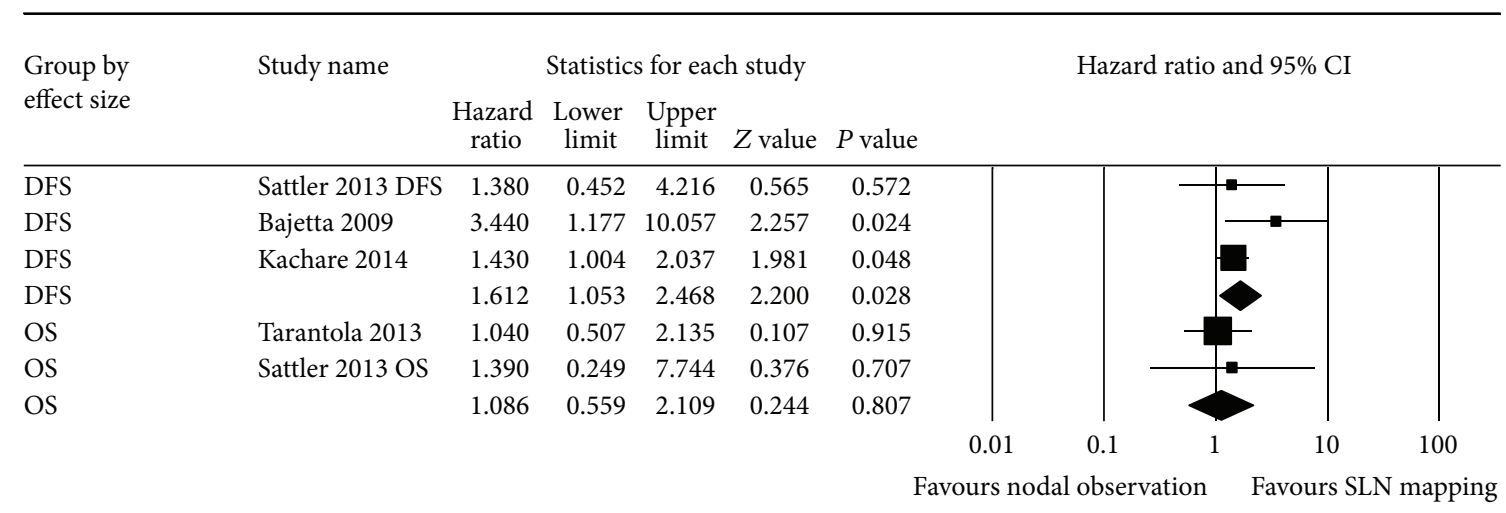

FIGURE 2: Forest plot of the hazard ratio (HR) of disease free survival (DFS) and overall survival (OS) for operative staging with SLN mapping versus nodal observation.

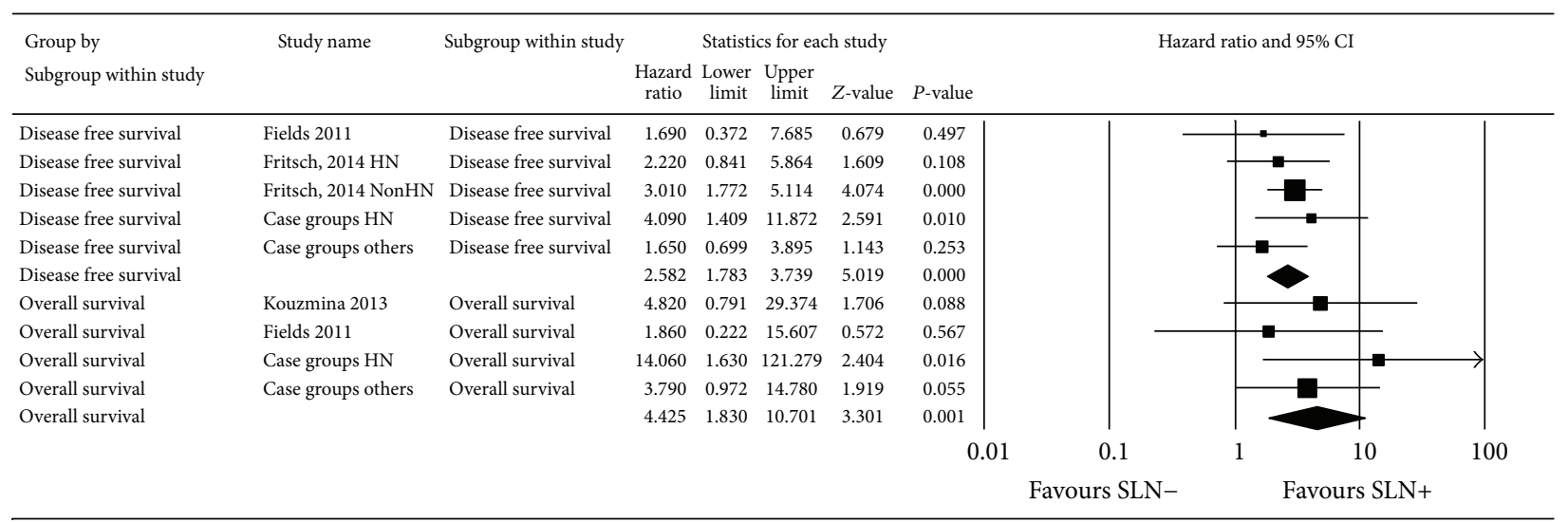

FIGURE 3: Forest plot of the hazard ratio (HR) of disease free survival (DFS) and overall survival (OS) for pathological SLN status.

of SLN status in MCC and (2) to assess the prognostic impact of SLN mapping versus other nodal treatments (mainly nodal observation) in MCC.

4.1. Prognostic Significance of SLN+ Status in MCC. MCC is a rare aggressive cutaneous tumor. Accurate staging of MCC patients is highly important for proper treatment planning. Radiological examinations such as PET imaging and CT have been used for this purpose with various results [50].

Positive lymph node disease at presentation is a strong indicator of poor outcome and reduces the 5-year survival rate to less than $50 \%$ [11].

Although medical literature is rich regarding SLN mapping in MCC [27, 33], studies with true survival analysis for the importance of SLN mapping are scarce. Overall, 3 studies had appropriate survival analyses and they were included in the current systematic review. We also gathered data of 172 cases from the medical literature and performed an individual patient meta-analysis.

Our meta-analysis showed that SLN status is a strong predictor of OS and DFS in MCC patients (pooled HR of 4.42 and 2.58 , resp.). In other words, SLN+ patients may suffer MCC- related death and recurrence 4.42 and 2.58 times more frequently per unit time than SLN- patients. It is worth mentioning that, only in the study of Fritsch et al. [16], SLN status was a statistically significant predictor of survival. The other two studies by Kouzmina et al. [19] and Fields et al. [15] showed statistically nonsignificant results despite HR >1 which denotes the lower MCC-related death or recurrence in the SLN- as compared to the SLN+ patients. The reasons of statistically nonsignificant results in these studies are most likely the low sample size and statistical power. Our meta-analysis by combining the results of individual studies increased the statistical power and yielded statistically significant results. Anyhow, the direction of effect size (HR) in the included studies is all the same and denotes the survival benefit of SLN- status. Location of the MCC is an important issue which has been brought up in the SLN mapping of MCC. Fritsch et al. [16] reviewed the information of SEER database and reported that SLN+ status was not an independent prognostic factor for predicting DFS. They attributed this finding to different lymphatic pathways and behavior in the head and neck area as compared to the other parts of the body $[16,51]$. However, our meta-analysis did not show any difference between head and neck and other parts of the body regarding prognostic value of SLN status in MCC patients. It seems that further studies with long follow-up are needed to further elucidate this issue. 


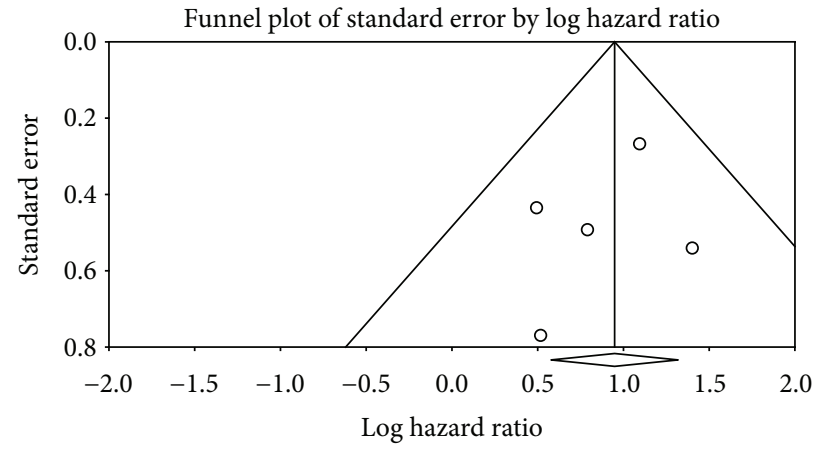

DFS

(a)

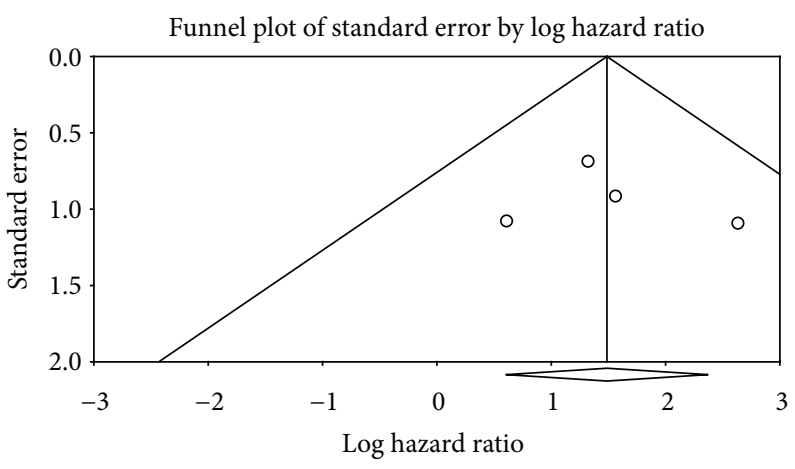

OS

(b)

FIGURE 4: Funnel plots of meta-analyses of pathological SLN status for DFS and OS.

4.2. Does SLN Mapping Actually Improves Survival in cNO MCC Patients? Bulk of medical literature is devoted to evaluate the accuracy of SLN mapping in MCC. However, we aimed to know if actually SLN mapping in cN0 MCC patients is associated with improved survival or not. Only four studies had enough survival analyses to answer the abovementioned clinical question and were included in the current systematic review. Three of the included studies compared operative nodal staging by SLN mapping with nodal observation only $[14,17,18]$. As shown in Table 1 , all of these studies showed survival benefit of the SLN mapping compared to observation strategy (HR > 1 for DFS). Our systematic review also supported the previous findings with pooled HR of 1.64 for DFS. The reason of this finding is most likely the early diagnosis of regional nodal involvement by SLN mapping which leads to start of the adjuvant treatments in earlier stages of the disease course providing a better DFS.

On the other hand the pooled HR for OS was not statistically significant ( $\mathrm{HR}=1.08, P=0.093)$. This is most likely due to the different design of Tarantola et al. study [3] as they reported the survival benefit of SLN mapping as compared to other nodal treatments (including regional nodal dissection, nodal radiation therapy, and observation) in cN0 MCC patients. Other nodal treatment strategies besides nodal observation can introduce a bias into the study as they can have survival benefit compared to nodal observation alone.

To sum up, it seems that operative nodal staging with SLN mapping provides survival benefit versus nodal observation in cN0 MCC patients. The survival benefit is mostly obvious for DFS. In order to evaluate the effect of SLN mapping on OS, larger studies with long follow-up are still needed.

4.3. Limitations. MCC is a rare tumor and many authors reported their experience in SLN mapping in this tumor with small sample size. In order to overcome this problem, we gathered the prognostic information in the literature performing survival analysis in the final sample of cases (172 patients). Although studies based on SEER database had very large sample size, the other included studies had relatively small sample size and this may limit the statistical power of our meta-analysis.

Comparison of SLN mapping with nodal observation in all but one of the included studies is another limitation of our study. Survival benefit of SLN mapping ought to be compared with other nodal treatment protocols such as prophylactic regional node dissection or irradiation. Thus far only Tarantola et al. study reported survival benefit of SLN mapping as compared to other nodal treatment methods and further multicenter large studies are needed in this regard.

The quality of the included studies can also be considered as a limitation of our study. As shown in Table 1, only two of the included studies reported the details of SLN mapping technique.

Publication bias is a major concern in all systematic reviews. We evaluated this bias by funnel plots and Egger's regression method. Although neither funnel plots nor Egger's test showed possible publication bias, the power of Egger's test is relatively low and we cannot rule out possible important publication bias in our systematic review.

\section{Conclusion}

SLN mapping can provide strong prognostic information regarding OS and DFS in cN0 MCC patients. SLN+ patients may suffer MCC-related death and recurrence more frequently per unit time than SLN- patients. Further multicenter studies with long follow-up are needed to evaluate the effect of the location of MCC.

More importantly, SLN mapping can improve DFS and possibly OS in cN0 MCC patients as compared to nodal observation. As MCC is a rare tumor, large multicenter prospective studies are still needed to validate the survival benefit of SLN mapping. Further studies ought to compare SLN mapping with other nodal treatment strategies such as prophylactic lymph node dissection and/or irradiation.

\section{Conflict of Interests}

The authors declare that there is no conflict of interests regarding the publication of this paper. 


\section{Acknowledgments}

This study is the result of a residency thesis under the approval number of 920574 . The study was financially supported by the Vice Chancellery of research of Mashhad University of Medical Sciences.

\section{References}

[1] R. Pink, J. Ehrmann, M. Molitor et al., "Merkel cell carcinoma. A review," Biomedical Papers, vol. 156, no. 3, pp. 213-217, 2012.

[2] I. Prieto Muñoz, J. Pardo Masferrer, J. Olivera Vegas, M. S. Medina Montalvo, R. Jover Díaz, and A. M. Pérez Casas, "Merkel cell carcinoma from 2008 to 2012: reaching a new level of understanding," Cancer Treatment Reviews, vol. 39, no. 5, pp. 421-429, 2013.

[3] T. I. Tarantola, L. A. Vallow, M. Y. Halyard et al., "Prognostic factors in Merkel cell carcinoma: analysis of 240 cases," Journal of the American Academy of Dermatology, vol. 68, no. 3, pp. 425432, 2013.

[4] V. A. Smith, E. R. Camp, and E. J. Lentsch, "Merkel cell carcinoma: identification of prognostic factors unique to tumors located in the head and neck based on analysis of SEER data," Laryngoscope, vol. 122, no. 6, pp. 1283-1290, 2012.

[5] H. M. Herbert, M. T. Sun, D. Selva et al., "Merkel cell carcinoma of the eyelid: management and prognosis," JAMA Ophthalmology, vol. 132, no. 2, pp. 197-204, 2014.

[6] A. Yiengpruksawan, D. G. Coit, H. T. Thaler, C. Urmacher, and W. K. Knapper, "Merkel cell carcinoma: prognosis and management," Archives of Surgery, vol. 126, no. 12, pp. 1514-1519, 1991.

[7] R. Sadeghi, H. Gholami, S. R. Zakavi, V. R. D. Kakhki, K. T. Tabasi, and S. Horenblas, "Accuracy of sentinel lymph node biopsy for inguinal lymph node staging of penile squamous cell carcinoma: systematic review and meta-analysis of the literature," Journal of Urology, vol. 187, no. 1, pp. 25-31, 2012.

[8] M. Hassanzade, M. Attaran, G. Treglia, Z. Yousefi, and R. Sadeghi, "Lymphatic mapping and sentinel node biopsy in squamous cell carcinoma of the vulva: systematic review and meta-analysis of the literature," Gynecologic Oncology, vol. 130, no. 1, pp. 237-245, 2013.

[9] M. Ansari, M. A. Rad, M. Hassanzadeh et al., "Sentinel node biopsy in endometrial cancer: systematic review and metaanalysis of the literature," European Journal of Gynaecological Oncology, vol. 34, no. 5, pp. 387-401, 2013.

[10] S. G. Gupta, L. C. Wang, P. F. Peñas, M. Gellenthin, S. J. Lee, and P. Nghiem, "Sentinel lymph node biopsy for evaluation and treatment of patients with Merkel cell carcinoma: the DanaFarber experience and meta-analysis of the literature," Archives of Dermatology, vol. 142, no. 6, pp. 685-690, 2006.

[11] K. Mehrany, C. C. Otley, R. H. Weenig, P. Kim Phillips, R. K. Roenigk, and T. H. Nguyen, "A meta-analysis of the prognostic significance of sentinel lymph node status in Merkel cell carcinoma," Dermatologic Surgery, vol. 28, no. 2, pp. 113-117, 2002.

[12] J. H. F. Shaw and E. Rumball, "Merkel cell tumour: clinical behaviour and treatment," British Journal of Surgery, vol. 78, no. 2, pp. 138-142, 1991.

[13] S. Hasan, L. Liu, J. Triplet, Z. Li, and D. Mansur, "The role of postoperative radiation and chemoradiation in merkel cell carcinoma: a systematic review of the literature," Frontiers in Oncology, vol. 3, article 276, 2013.
[14] S. D. Kachare, J. H. Wong, N. A. Vohra, E. E. Zervos, and T. L. Fitzgerald, "Sentinel lymph node biopsy is associated with improved survival in merkel cell carcinoma," Annals of Surgical Oncology, vol. 21, no. 5, pp. 1624-1630, 2014.

[15] R. C. Fields, K. J. Busam, J. F. Chou et al., "Recurrence and survival in patients undergoing sentinel lymph node biopsy for Merkel cell carcinoma: analysis of 153 patients from a single institution," Annals of Surgical Oncology, vol. 18, no. 9, pp. 25292537, 2011.

[16] V. A. Fritsch, E. R. Camp, and E. J. Lentsch, "Sentinel lymph node status in Merkel cell carcinoma of the head and neck: not a predictor of survival," Head and Neck, vol. 36, no. 4, pp. 571579, 2014.

[17] E. Bajetta, L. Celio, M. Platania et al., "Single-institution series of early-stage merkel cell carcinoma: long-term outcomes in 95 patients managed with surgery alone," Annals of Surgical Oncology, vol. 16, no. 11, pp. 2985-2993, 2009.

[18] E. Sattler, T. Geimer, I. Sick et al., "Sentinel lymph node in Merkel cell carcinoma: to biopsy or not to biopsy?" Journal of Dermatology, vol. 40, no. 5, pp. 374-379, 2013.

[19] M. Kouzmina, J. Leikola, T. Böhling, and V. Koljonen, "Positive sentinel lymph node biopsy predicts local metastases during the course of disease in Merkel cell carcinoma," Journal of Plastic Surgery and Hand Surgery, vol. 47, no. 2, pp. 139-143, 2013.

[20] M. K. Parmar, V. Torri, and L. Stewart, "Extracting summary statistics to perform meta-analyses of the published literature for survival endpoints," Statistics in Medicine, vol. 17, no. 24, pp. 2815-2834, 1998.

[21] http://www.cebm.net/index.aspx?o=5987 .

[22] R. DerSimonian and N. Laird, "Meta-analysis in clinical trials," Controlled Clinical Trials, vol. 7, no. 3, pp. 177-188, 1986.

[23] M. Egger, G. D. Smith, M. Schneider, and C. Minder, "Bias in meta-analysis detected by a simple, graphical test," British Medical Journal, vol. 315, no. 7109, pp. 629-634, 1997.

[24] S. E. Ames, D. N. Krag, and M. S. Brady, "Radiolocalization of the sentinel lymph node in Merkel cell carcinoma: a clinical analysis of seven cases," Journal of Surgical Oncology, vol. 67, no. 4, pp. 251-254, 1998.

[25] N. Wasserberg, J. Schachter, M. D. Eyal Fenig, M. Feinmesser, and H. Gutman, "Applicability of the sentinel node technique to Merkel cell carcinoma," Dermatologic Surgery, vol. 26, no. 2, pp. 138-141, 2000.

[26] L. K. E. Rodrigues, S. P. L. Leong, M. Kashani-Sabet, and J. H. Wong, "Early experience with sentinel lymph node mapping for Merkel cell carcinoma," Journal of the American Academy of Dermatology, vol. 45, no. 2, pp. 303-308, 2001.

[27] D. Pan, D. Narayan, and S. Ariyan, "Merkel cell carcinoma: five case reports using sentinel lymph node biopsy and a review of 110 new cases," Plastic and Reconstructive Surgery, vol. 110, no. 5, pp. 1259-1265, 2002.

[28] L. D. Su, L. Lowe, C. R. Bradford, A. I. Yahanda, T. M. Johnson, and V. K. Sondak, "Immunostaining for cytokeratin 20 improves detection of micrometastatic Merkel cell carcinoma in sentinel lymph nodes," Journal of the American Academy of Dermatology, vol. 46, no. 5, pp. 661-666, 2002.

[29] A. Blom, F. Kolb, J. Lumbroso et al., "Significance of sentinel lymph node biopsy in Merkel cell carcinoma. Analysis of II cases," Annales de Dermatologie et de Venereologie, vol. 130, no. 4, pp. 417-422, 2003.

[30] J. C. Alex, "The application of sentinel node radiolocalization to solid tumors of the head and neck: a 10-year experience," Laryngoscope, vol. 114, no. 1, pp. 2-19, 2004. 
[31] C. E. Schmalbach, L. Lowe, T. N. Teknos, T. M. Johnson, and C. R. Bradford, "Reliability of sentinel lymph node biopsy for regional staging of head and neck Merkel cell carcinoma," Archives of Otolaryngology - Head and Neck Surgery, vol. 131, no. 7, pp. 610-614, 2005.

[32] F. J. Civantos, F. L. Moffat, and W. J. Goodwin, "Lymphatic mapping and sentinel lymphadenectomy for 106 head and neck lesions: contrasts between oral cavity and cutaneous malignancy," Laryngoscope, vol. 116, supplement 109, no. 3, pp. $1-15,2006$.

[33] S. Maza, U. Trefzer, M. Hofmann et al., "Impact of sentinel lymph node biopsy in patients with Merkel cell carcinoma: results of a prospective study and review of the literature," European Journal of Nuclear Medicine and Molecular Imaging, vol. 33, no. 4, pp. 433-440, 2006.

[34] J. Ortin-Perez, M. C. van Rijk, R. A. Valdes-Olmos et al., "Lymphatic mapping and sentinel node biopsy in Merkel's cell carcinoma," European Journal of Surgical Oncology, vol. 33, no. 1, pp. 119-122, 2007.

[35] V. Koljonen and S. Suominen, "Sentinel node biopsy in local anaesthesia in patients with head and neck Merkel cell carcinoma," European Journal of Plastic Surgery, vol. 30, no. 5, pp. 205-210, 2008.

[36] E. Migliano, C. Monarca, M. Tedesco, M. I. Rizzo, and S. Bucher, "Merkel cell carcinoma and sentinel lymph node dissection: nine cases report," Il Giornale di Chirurgia, vol. 29, no. 1-2, pp. 28-32, 2008.

[37] L. R. Ramón, F. A. Javier, M. Roberto et al., "Merkel cell carcinoma of the head and neck: report of seven cases," Medicina Oral, Patologia Oral y Cirugia Bucal, vol. 13, no. 6, pp. E390E394, 2008.

[38] Y. Shnayder, D. T. Weed, D. J. Arnold et al., "Management of the neck in Merkel cell carcinoma of the head and neck: university of Miami experience," Head and Neck, vol. 30, no. 12, pp. 15591565, 2008.

[39] T. Maalouf, J. L. George, L. Geoffrois, P. Olivier, and G. Dolivet, "Sentinel lymph node biopsy for lymphophilic conjunctival and eyelid tumours: apropos of 8 cases," Revue de Laryngologie Otologie Rhinologie, vol. 130, no. 4-5, pp. 231-234, 2009.

[40] R. Kamo, A. Kumei, S. Ueoku, N. Kusutani, J. Sowa, and M. Ishii, "Importance of sentinel lymph node biopsy in Merkel cell carcinoma," Journal of Dermatology, vol. 37, no. 12, pp. 10681070, 2010.

[41] J. Howle and M. Veness, "Sentinel lymph node biopsy in patients with Merkel cell carcinoma: an emerging role and the Westmead hospital experience," Australasian Journal of Dermatology, vol. 53, no. 1, pp. 26-31, 2012.

[42] M. L. Matthey-Gie, A. Boubaker, I. Letovanec, N. Demartines, and M. Matter, "Sentinel lymph node biopsy in nonmelanoma skin cancer patients," Journal of Skin Cancer, vol. 2013, Article ID 267474, 8 pages, 2013.

[43] A. Marchesi, P. Camillo Parodi, M. Brioschi, G. Sileo, M. Marchesi, and L. Vaienti, "Giant merkel cell carcinoma of the lower limb: case report and review of the literature," Journal of Cutaneous Medicine and Surgery, vol. 17, pp. 1-5, 2013.

[44] N. C. Zeitouni, R. T. Cheney, and M. D. Delacure, "Lymphoscintigraphy, sentinel lymph node biopsy, and Mohs micrographic surgery in the treatment of merkel cell carcinoma," Dermatologic Surgery, vol. 26, no. 1, pp. 12-18, 2000.
[45] I. Düker, H. Starz, D. Bachter, and B.-R. Balda, "Prognostic and therapeutic implications of sentinel lymphonodectomy and Sstaging in merkel cell carcinoma," Dermatology, vol. 202, no. 3, pp. 225-229, 2001.

[46] G. Giannotti, D. Lazzeri, P. Viacava et al., "Merkel cell carcinoma of the lower extremity: report of four cases and new considerations," Annals of Plastic Surgery, vol. 62, no. 1, pp. 83-86, 2009.

[47] M. Mehrabibahar, M. N. Forghani, B. Memar et al., "Sentinel lymph node biopsy in melanoma patients: an experience with Tc-99m antimony sulfide colloid," Iranian Journal of Nuclear Medicine, vol. 18, no. 1, pp. 1-6, 2010.

[48] M. Aliakbarian, B. Memar, A. Jangjoo et al., "Factors influencing the time of sentinel node visualization in breast cancer patients using intradermal injection of the radiotracer," The American Journal of Surgery, vol. 202, no. 2, pp. 199-202, 2011.

[49] K. M. McMasters, D. S. Reintgen, M. I. Ross et al., "Sentinel lymph node biopsy for melanoma: controversy despite widespread agreement," Journal of Clinical Oncology, vol. 19, no. 11, pp. 2851-2855, 2001.

[50] G. Treglia, R. Sadeghi, S. Annunziata et al., "Diagnostic performance of Fluorine-18-Fluorodeoxyglucose positron emission tomography for the diagnosis of osteomyelitis related to diabetic foot: a systematic review and a meta-analysis," Foot, vol. 23, no. 4, pp. 140-148, 2013.

[51] S. J. Miller, M. Alam, J. Andersen et al., "Merkel cell carcinoma: clinical practice guidelines in oncology," JNCCN Journal of the National Comprehensive Cancer Network, vol. 4, no. 7, pp. 704712, 2006. 


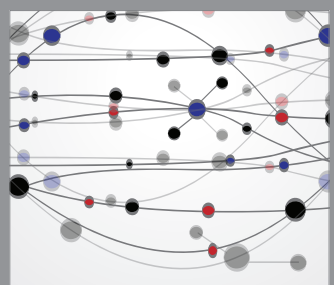

The Scientific World Journal
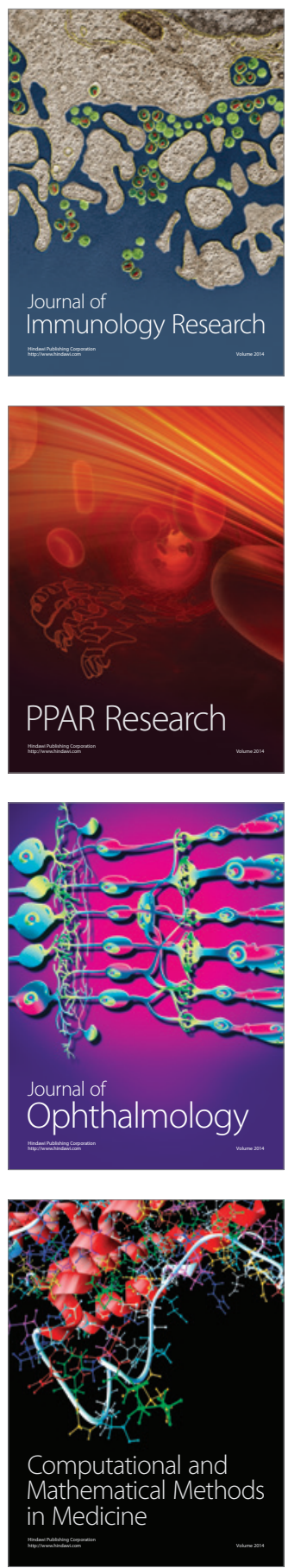

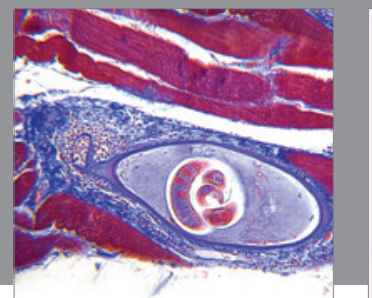

Gastroenterology

Research and Practice
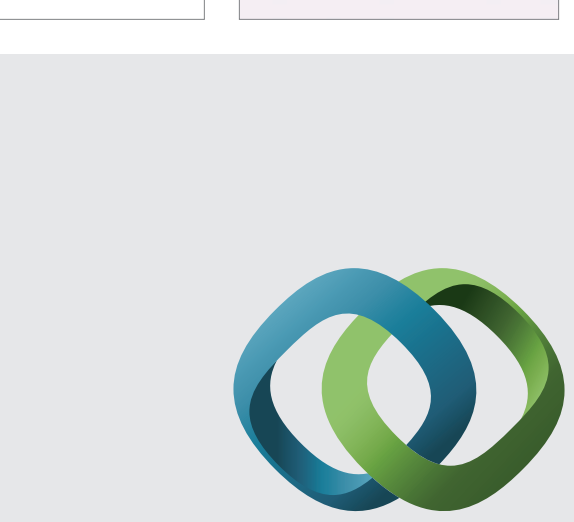

\section{Hindawi}

Submit your manuscripts at

http://www.hindawi.com
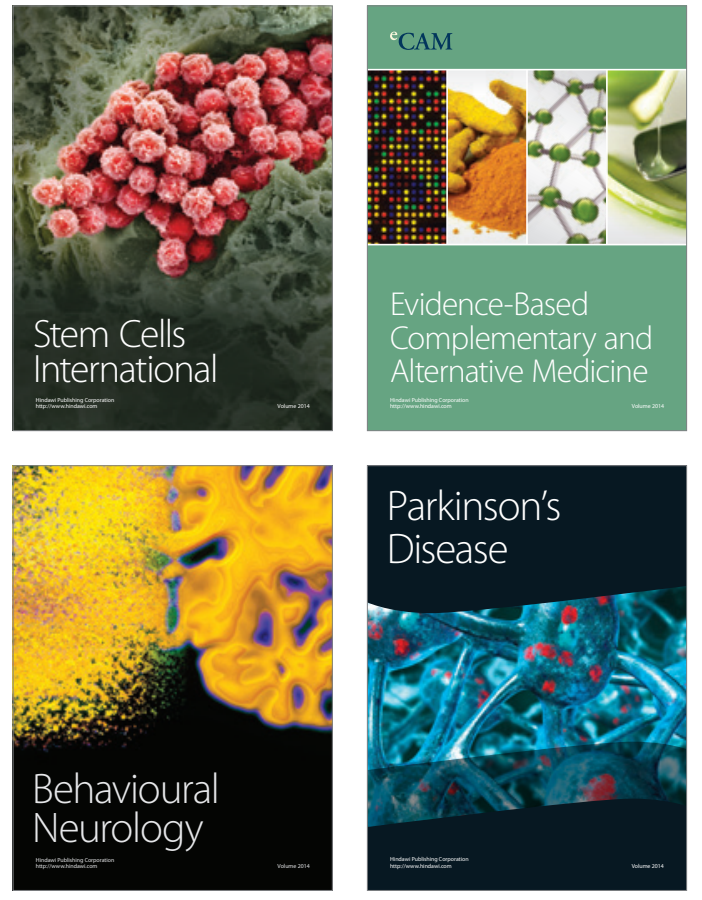
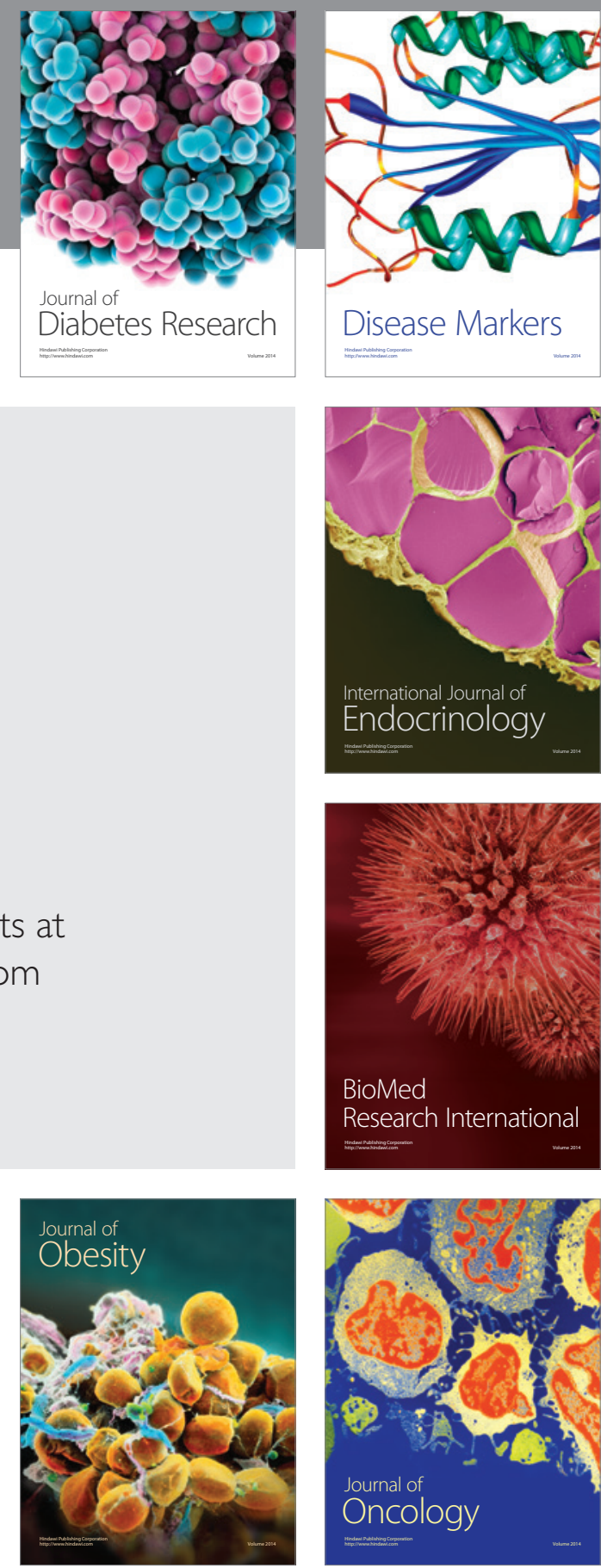

Disease Markers
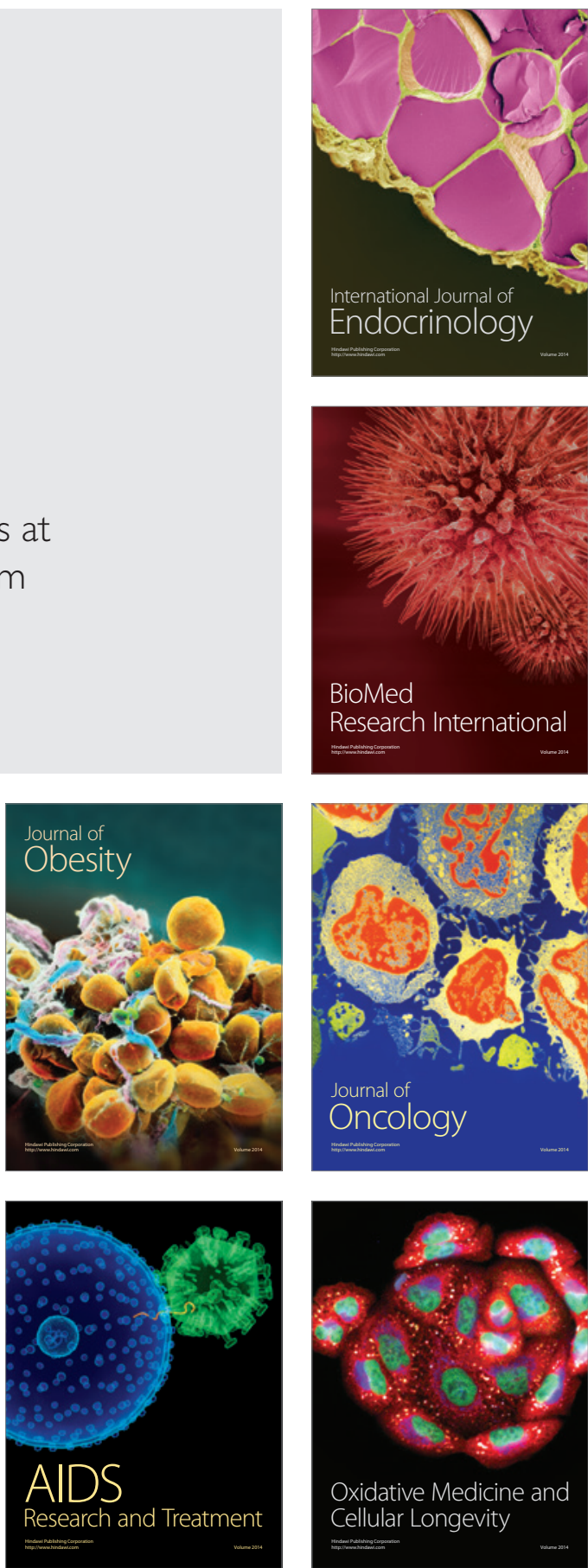